\title{
Social defeat alters the acquisition of cocaine self-administration in rats: role of individual differences in cocaine-taking behavior
}

Received: 30 May 2001 / Accepted: 25 August 2001 / Published online: 1 November 2001

(C) Springer-Verlag 2001

\begin{abstract}
Rationale: It is known that social defeat can modulate cocaine self-administration. However, it is unclear whether this psychosocial stressor affects drugtaking behavior to the same extent across all individual animals, particularly those with differing propensities to self-administer psychostimulants. Objective: This study examined the effect of social defeat on cocaine selfadministration in animals that differ in novelty-seeking behavior that predicts differences in drug self-administration. Methods: Male Sprague-Dawley rats were first classified into high-responder (HR) and low-responder (LR) groups. HR and LR rats were categorized based on their locomotor activity in a novel environment, with HR rats exhibiting higher locomotor activity than LR rats. Then, male rats were exposed on four occasions to an aggressive Long Evans male rat over the course of 4 days. Control rats were not exposed to the social defeat. All rats were subsequently implanted with jugular catheters and 3 days later placed into the self-administration box to study the acquisition of cocaine selfadministration ( $0.25 \mathrm{mg}$ per infusion). Results: HR nondefeated animals self-administered more cocaine than the LR non-defeated animals. Following social defeat, the acquisition of cocaine self-administration is significantly delayed in HR rats and enhanced in LR rats. Conclusion: The unique patterns of responsiveness in the HR and LR animals suggest that social defeat plays a role of equalizer of individual differences in drugtaking behavior.
\end{abstract}

Keywords Cocaine $\cdot$ High responder $\cdot$ Intravenous self-administration - Social defeat $\cdot$ Low responder . Novelty

M. Kabbaj ( $)$ C.S. Norton · S. Kollack-Walker

S.J. Watson - H. Akil

Mental Health Research Institute, The University of Michigan,

205 Zina Pitcher Place, Ann Arbor, MI 48109-0720, USA

Tel.: +1-734-9362034, Fax: +1-734-6474130

T.E. Robinson

Department of Psychology, University of Michigan,

Ann Arbor, MI 48109, USA

\section{Introduction}

In animal studies, the interplay between stress and the actions of drugs of abuse has become a recent focus of investigation (Piazza and Le Moal 1997; Piazza et al. 1998). For example, various stressors increase the locomotor effects of psychostimulants and opioids (Robinson and Becker 1986; Kalivas and Stewart 1991; Badiani and Stewart 1993), and repeated exposure to stress can sensitize an animal to a subsequent stressor and/or drug exposure (Sorg and Kalivas 1991). Stress can also influence drug self-administration (SA) behavior. Physical stressors, such as intermittent tail pinch and unpredictable foot shock, enhance the acquisition of cocaine SA (Piazza et al. 1990; Goeders and Guerin 1994), as do social stressors, such as isolation (Kosten et al. 2000) and social defeat (Haney et al. 1995; Miczek et and Mutscler 1996).

While the interplay between stress and drug-taking behavior is now well established, it is unclear whether this interaction is uniform in all individuals or whether it is modulated by differences in responsiveness to stress, drugs, or both. In this study, we focus on the effect of a psychosocial stressor on individual differences in drugtaking behavior. One form of psychosocial stress is social defeat, "an environmental stressor which offers the advantage of ecological and ethological validity" (Tidey and Miczek 1997). The model of social defeat that we used is initiated when a subordinate male rat replaces the female rat in the home cage of an aggressive dominant male. When placed in this situation, the intruder male behaves submissively, exhibits a rise in the levels of the stress hormones corticosterone (CORT) and adrenocorticotropic hormone (ACTH) (Marti-Carbonell et al. 1992) and shows an increase in accumbal and prefrontal dopamine (DA) release (Miczek et al. 1994; Tidey and Miczek 1996). Social defeat also increases cocaine SA when tested 1 day, 7 days or even 30 days after defeat (Tidey and Miczek 1997; Covington and Miczek 2000).

While highly interesting, the above-mentioned studies have not systematically investigated the role of individual differences on the interplay between social defeat and 
drug-taking behavior. Certain genetic and environmental factors may render particular individuals more vulnerable to the effects of stressful situations. This vulnerability may be apparent in a number of behavioral manifestations possibly including drug-taking behavior. Furthermore, the drug-taking behavior itself may have somewhat different triggers in different animals. It is therefore reasonable to examine individual differences in the effect of social stress on drug SA.

A body of work describes a model in which animals exhibit significant individual differences in the acquisition of stimulant SA (Piazza et al. 1989, 1991). Outbred rats can be differentiated based on their locomotor activity in a novel environment. Some rats display a great deal of exploratory activity when placed in a new environment and are termed high responders (HRs). Other rats exhibit a lower rate of exploration of a new surrounding and are termed low responders (LRs). HR rats learn to selfadminister psychostimulants faster than LR rats (Piazza et al. 1989). Our own unpublished studies on amphetamine SA in HR versus LR rats suggest that, with enough time, both groups reach similar rates of drug taking and that the main difference between the groups resides in the rate of acquisition, rather than in the maintenance phase (but also see Piazza et al. 2000). Interestingly, HR and LR rats have different neurochemical, endocrine, and behavioral responses to physical (Piazza et al. 1998) and social (Kabbaj et al. 2000) stressors. It is unknown whether social defeat alters the acquisition phase of cocaine SA in HR and LR rats; thus, the present study investigated the possible differential effect of social defeat on the acquisition of cocaine SA in HR and LR rats.

\section{Materials and methods}

Subjects

Fifty-six male Sprague-Dawley rats from Charles River (Wilmington, Mass.), weighing 250-300 $\mathrm{g}$ at the beginning of the study and $400-450 \mathrm{~g}$ at the end of the study, were used in the SA experiment. Those animals were housed three per cage $(43 \times 21.5 \times 25.5-\mathrm{cm}$ Plexiglass cages) and were singly housed following social defeat. In addition, 20 vasectomized male Long-Evans rats weighing 500-550 g were housed with female Long-Evans rats. These males were used as resident attackers and were chosen from an original group of 26 rats for their consistent aggressive behavior. From the 56 Sprague-Dawley rats, 10 rats (5 HR and 5 LR) were used to test the aggressive behavior of Long Evans rats. This test consisted of a single 5-min encounter. All animals were housed on an 11-h/13-h light/dark cycle (lights on at 2100 hours), and food and water were available ad libitum.

All experiments were conducted in accordance with the guidelines of the animal ethics committee at the University of Michigan following the Guide for the Care and Use of Laboratory Animals (National Research Council 1996).

\section{Experimental design}

This study was completed using two cohorts of animals that were run at a 3-month interval. Each cohort contained the same experimental groups (HR defeated, LR defeated, HR non-defeated, and LR non-defeated).
Sprague-Dawley rats were acclimated to the animal colony for 1 week and then screened for their locomotor response in a novel environment by placing them in a $43 \times 21.5 \times 24.5-\mathrm{cm}$ (high) clear acrylic activity monitor. Locomotor activity was monitored by means of two banks of photocells connected to a microprocessor. This 60-min test was conducted during a light portion of the light/dark cycle (2100-2400 hours). After assessing that the locomotor activity has a normal distribution, the median score for all rats was calculated, and rats that had a locomotor score above the median were deemed HRs, while those with a score below the median were deemed LRs.

The day after the locomotion test, equal numbers of HR and LR animals were assigned to one of two groups: socially defeated or control. Following the 4 days of social defeat or handling, the rats were implanted with jugular catheters. Cocaine SA was initiated 3 days later and was continued for a period of 9 days.

\section{Social defeat}

The social stress paradigm consisted of four encounters with an aggressive Long-Evans male rat. Each Long-Evans male rat was vasectomized and housed with a normal cycling female rat to enhance territorial behavior and aggressiveness. Prior to each agonistic interaction, the resident's mate was transferred to another cage. A Sprague-Dawley male rat was then placed as an intruder into the resident male's cage, and the rats were allowed 5 min to interact. After this initial $5 \mathrm{~min}$, the rats had an extra $10 \mathrm{~min}$ to interact, and, if the fighting became too intense, the intruder rat was transferred to a protective metal cage and placed back into the resident's home cage, which allowed for intense visual, auditory, and olfactory interactions (Miczek et al. 1991). This cage was of sufficient size to allow animals to move freely $(10 \times 10 \times 15 \mathrm{~cm})$. Intruder males exhibited the following behaviors during an agonistic interaction: immobility (four paws on ground, orienting toward resident), escape (fleeing the resident), crouching (four paws on ground, not orienting toward resident), defensive upright (standing erect with forepaws extended), walking, rearing and grooming (Miczek 1979; Haney and Miczek 1993; Miczek and Mutschler 1996). The agonistic interactions occurred under redlight conditions, during a portion of the dark phase of the light/dark cycle (1100-1400 hours). During the first $5 \mathrm{~min}$ of the four defeat occasions, the rat's defensive behavior was videotaped and later quantified.

\section{Cocaine SA}

Following the 4 days of exposure to social stress or the control conditions, intracardiac catheters were inserted through the external jugular vein, passed under the skin, and fixed in the mid-scapular region. Cocaine SA testing began 3 days later. Rats were trained under a fixed-ratio 1 (FR1) schedule of SA. For each test session, rats were connected to the tether and placed in the SA boxes, and lever pressing was measured over the course of a 2-h test session. Each operant conditioning chamber was a sound-attenuating chamber that shields animals from extraneous disturbances. White noise was supplied in each chamber using a ventilating fan. Each operant conditioning chamber contained two levers. Presses on one lever (the active lever) resulted in an infusion of cocaine through the catheter $(0.25 \mathrm{mg}$ per infusion). Cocaine was injected in a volume of $0.1 \mathrm{ml}$ per infusion at a rate of $1.5 \mathrm{ml} / \mathrm{min}$. For $20 \mathrm{~s}$ immediately following depression of the active lever, the stimulus light located above the lever was lit. During this 20 -s period, presses on the active lever were recorded but did not lead to further infusions. Presses on the inactive lever were also recorded but were without consequences. A criterion for the acquisition of cocaine SA was established a priori: a rat was deemed as having undergone cocaine SA when it received ten or more infusions of cocaine per session on two consecutive days during the 9 days of SA. Based on this criterion three HR defeated, two HR non-defeated, two LR defeated and two LR non-defeated were excluded from the experiment because they did not reach the criterion of acquisition. Following each SA session, rats were returned to their home cages and their catheters were flushed with $0.1 \mathrm{ml}$ heparin solution. Every 4 days, the patency 

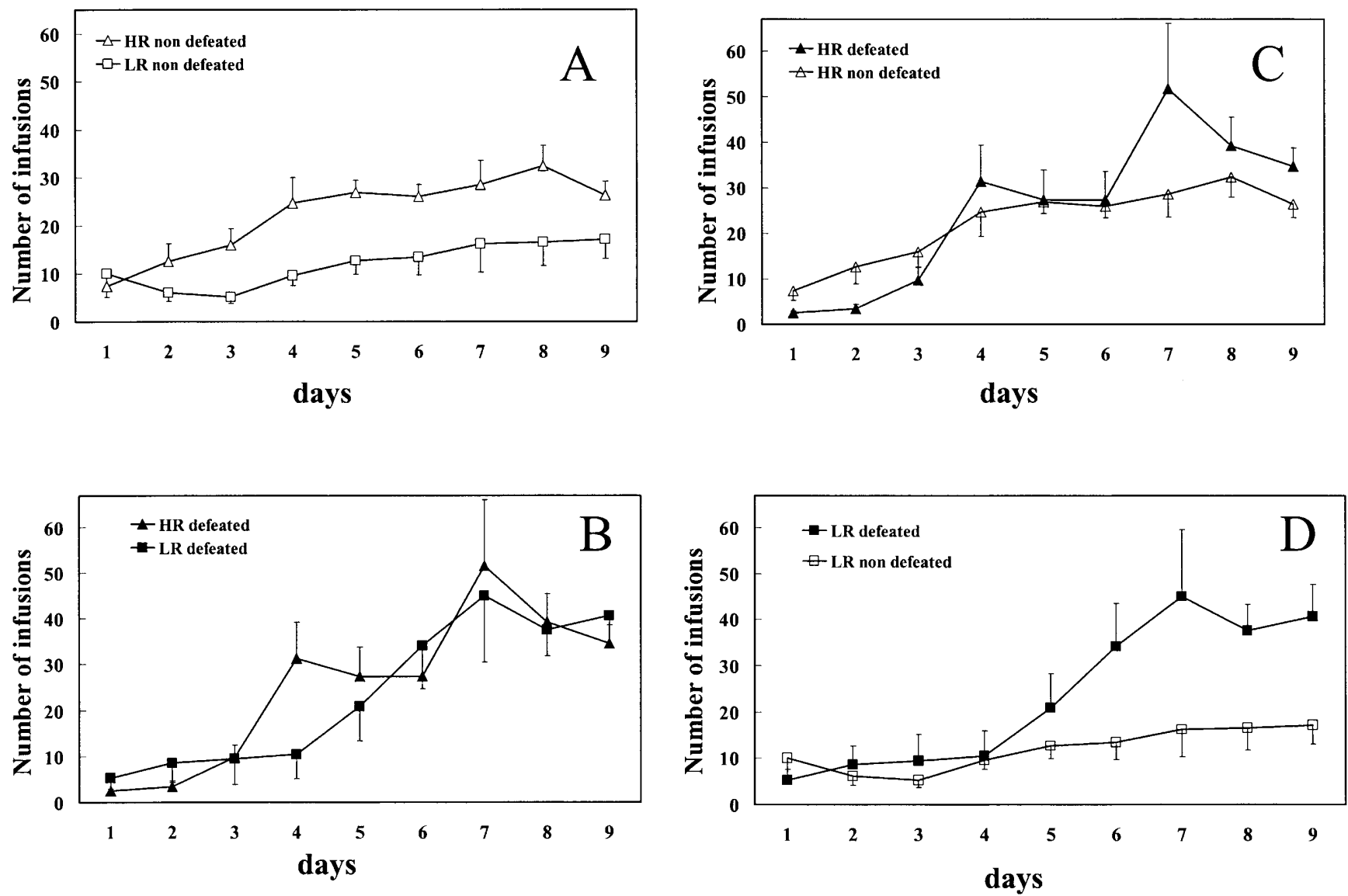

Fig. 1 A High responder (HR) non-defeated $(n=8)$ and low responder (LR) non-defeated $(n=8)$ cocaine intake $(0.25 \mathrm{mg} / \mathrm{kg})$ during 9 days of self-administration. B HR defeated $(n=8)$ and LR defeated $(n=8)$ cocaine intake $(0.25 \mathrm{mg} / \mathrm{kg})$ during 9 days of selfadministration. C HR defeated $(n=8)$ and HR non-defeated $(n=8)$ cocaine intake $(0.25 \mathrm{mg} / \mathrm{kg})$ during 9 days of self-administration. D LR defeated $(n=8)$ and LR non-defeated $(n=8)$ cocaine intake $(0.25 \mathrm{mg} / \mathrm{kg})$ during 9 days of self-administration. All results are expressed as mean \pm SEM

of the catheters was determined by injecting $0.1 \mathrm{ml}$ pentobarbital into the catheter. Rats that did not lose muscle tone within 2-3 s were excluded from the experiment. Based on this criterion, five rats were excluded from the experiment.

\section{Statistical analysis}

A two-way, repeated-measures analysis of variance (ANOVA) was used to test differences in the number of infusions between HR/LR and defeat/non-defeat groups across the 9 days of cocaine SA. Fisher post-hoc analysis followed this ANOVA.

Behavioral encounters during defeat were also analyzed with two-way, repeated-measures ANOVA. The between-subjects factors in this analysis were individual differences factor (HR/LR) and the stress factor defeat/non-defeat. The within-subject factor was the 4 days of encounter with the aggressive male.

\section{Results}

Social defeat

All Sprague Dawley rats were defeated at least three times. A rat was considered not defeated if it was not

Table 1 Frequency of the defensive behaviors expressed by highresponder (HR; $n=8)$ and low-responder $(\mathrm{LR} ; n=8)$ rats during their encounter with aggressive Long Evans male rats. Results are expressed as mean \pm SEM

\begin{tabular}{llrrr}
\hline & \multicolumn{1}{l}{ Day 1} & \multicolumn{1}{c}{ Day 2} & \multicolumn{1}{l}{ Day 3} & \multicolumn{1}{c}{ Day 4 } \\
\hline HR supine & $1.37 \pm 0.53$ & $2.37 \pm 0.77$ & $3.87 \pm 1.59$ & $3 \pm 1.28$ \\
LR supine & $2.12 \pm 0.47$ & $3.5 \pm 1.03$ & $2.12 \pm 0.58$ & $2.62 \pm 0.46$ \\
HR escape & $2 \pm 0.92$ & $2 \pm 0.42$ & $1.12 \pm 0.35$ & $1.62 \pm 0.56$ \\
LR escape & $1.75 \pm 0.55$ & $1.62 \pm 0.49$ & $1 \pm 0.42$ & $1.37 \pm 0.49$ \\
HR upright & $8.62 \pm 1.46$ & $8.87 \pm 1.18$ & $9 \pm 0.88$ & $6.87 \pm 1.43$ \\
LR upright & $8.25 \pm 0.95$ & $7.62 \pm 1.14$ & $7.5 \pm 1.28$ & $7.87 \pm 0.51$ \\
\hline
\end{tabular}

attacked and did not show any behavioral reactivity (supine, escape, upright) to the aggressor during the $15 \mathrm{~min}$ of encounter. No differences in the behavioral response to attack were apparent between the HR and LR groups. This is demonstrated by finding no difference between HR and LR rats for supine behavior, defensive upright, and escape behavior (Table 1).

\section{Effects of defeat on cocaine SA in HR and LR rats}

When non-defeated, HR rats showed greater cocaine intake than LR rats (group effect, $F_{1,14}=12.99, P=0.002$ ). This difference was present throughout the cocaine SA experiment (group $\times$ days interaction, $F_{8,112}=1.46$, $P=0.17$; Fig. 1A. Interestingly, after defeat, HR rats no 
longer differed from LR rats with regard to cocaine intake (Fig. 1B).

Examination of the findings also revealed that defeat stress inhibited HR cocaine self-infusions during the first 3 days of cocaine SA. This is supported by a no group effect $\left(F_{1,14}=0.68, P=0.68\right)$ and a tendency toward significance of the interaction group effect $\times$ days $\left(F_{8,112}=1.78\right.$, $P=0.08$ ). Post-hoc analyses show that, relative to HR non-defeated rats, HR defeated rats received significantly fewer infusions on day 1 and day $2(P<0.05)$ and exhibited a tendency for fewer infusions on day $3(P=0.1)$. Beyond that time point, HR defeated and non-defeated animals received an equivalent number of similar infusions on days 4-9 ( $P>0.05$; Fig. 1C).

By contrast, defeat stress increased cocaine intake in LR rats especially at a later time period. This effect is demonstrated by the presence of a group effect $\left(F_{1,14}=5.59, P=0.03\right)$ and the presence of a group $\times$ days interaction $\left(F_{8,112}=2.65, \quad P=0.01\right)$. Post-hoc analysis shows that LR defeated and LR non-defeated rats had a similar number of infusions on days 1-5 $(P>0.05)$. However, on days 6-9, LR defeated rats showed greater cocaine intake than LR non-defeated rats $(P<0.05$; Fig. 1D).

The number of presses on the active lever was significantly higher than on the inactive lever on every day of SA $(P<0.001)$. The number of presses on the inactive levers did not differ among the four groups $(P>0.5)$.

\section{Discussion}

The present study showed that HR non-defeated animals quickly underwent cocaine SA, when compared with LR non-defeated rats. This effect was delayed in HR rats exposed to social defeat who reached the criterion of cocaine SA by day 4 . Furthermore, compared with HR defeated rats, HR non-defeated animals received more cocaine during the first 3 days of SA. However, these same groups did not differ with regard to cocaine intake in the remaining days of SA.

The LR non-defeated and the LR defeated rats reached the criterion of cocaine SA by day 4, exactly at the same time point as the HR defeated rats. Additionally, although both LR groups consumed the same amount of drug on the first 5 days of testing, the LR defeated group consumed significantly more cocaine in the last 4 days of SA.

Possibly the most graphic demonstration of the differential effect of social defeat in the two groups of animals can be seen by comparing them under the stress versus the non-stress conditions. In the control, non-defeated conditions, there was a profound difference in cocaine SA between them, with HR animals consuming much more that LR animals. However, in the defeated animals, this inter-group difference disappeared completely and the animals appeared indistinguishable. In that regard, social defeat, by producing differential effects on the two groups, acted as an equalizer of initial individual differences in drug-taking behavior.

An increase in cocaine SA in socially defeated male rats has been previously reported. However, this effect of the social stressors on drug-taking behavior was not apparent in the first 2 days of SA (Haney et al. 1995). We found similar results when considering individual differences, as the inhibition was only apparent in the HR group and the later activation in both HR and LR animals, as HR defeated and LR defeated rats were taking more infusions of cocaine on days 4-9 compared with days 1-3. Tidey and Miczek (1997) demonstrated that socially defeated rats have an increased rate of cocaine acquisition relative to their non-defeated counterparts (Tidey and Miczek 1997). The present studies also found that defeat increased cocaine SA, but this was only evident in the LR group.

HR animals may be uniquely sensitive to social stress, including social isolation (Kabbaj et al. 2000). Such stressors tend to inhibit their novelty seeking behavior, rendering them more like LRs. A similar effect was seen in the present study where HR defeated animals resembled LR animals during the first few days of SA post-stress. Part of the differential effect of social stress on cocaine SA may be mediated by circulating glucocorticoids. There is excellent evidence that an optimal level of glucocorticoids may be necessary for the SA of psychostimulants (Goeders and Guerin 1996). Furthermore, there is strong evidence that HR and LR animals differ in terms of circulating glucocorticoids following stress (Piazza et al. 1989). Our own work has demonstrated that the pattern of gene expression of brain stress-related genes is significantly different in HR and LR animals. The pattern in the HRs, including high levels of gene expression of corticotropic releasing hormone in the paraventricular nucleus of the hypothalamus and low levels of glucocorticoid receptors in the hippocampus, favors larger and more prolonged stress responses in HR rats (Kabbaj et al. 2000). The difference in CORT levels in the HR and LR rats may mediate the differential rate of acquisition of drug SA. More importantly, it may also mediate, at least in part, the differential effects of social defeat on drug taking. While the levels of circulating glucocorticoids may be optimal for favoring drug acquisition in the HR non-defeated animals, they may readily exceed this optimal range following social defeat, thereby inhibiting SA for several days. Presumably, levels return to a more optimal range following a delay, and SA is resumed. By contrast, social stress may produce sufficient hypothalamic-pituitary-adrenal axis activation in the LR animals that it alters gene expression in their brain stress circuitry, rendering them more like HRs and leading them after several days to increased drug taking. These specific hypotheses are readily testable by monitoring the levels and time course of both circulating glucocorticoids and stress-related gene expression at different time points following the social stressor.

Similarly, the differential effects of social defeat may relate to anxiety. Although cocaine is a powerful 
reinforcer (Roberts et al. 1989; Wise 1996; Koob et al. 1997), it also increases anxiety-like behavior (Wood and Lal 1987; Ettenberg and Geist 1991; Rogerio and Takahashi 1992; Sarnyai 1998). Miczek et al. (1999) have shown that some animals generalize amphetamine and cocaine "cues" to social defeat stress, which may be relevant to anxiety-like effects of stimulants (Miczek et al. 1999). In fact, rats with high exploratory activity (like HR rats) are especially sensitive to cocaine's anxiogenic effects (Rogerio and Takahashi 1992). It is tempting to hypothesize that a high level of cocaineinduced anxiety could inhibit SA. Additionally, HR rats exposed to social defeat may be more susceptible to the anxiogenic effects of cocaine. This may account for the delayed acquisition of cocaine SA when compared with HR non-defeated controls. Conversely, LR animals may be inherently susceptible to the anxiogenic effects of cocaine, inhibiting them from obtaining substantial amounts of drug (as seen in the LR non-defeated group). At some point, HR rats may become either tolerant to the anxiogenic effects of cocaine and/or more vulnerable to the reinforcing effects of the drug, resulting in an initiation of cocaine SA. Upon repeated exposure to low doses of cocaine, LR rats, having experienced social defeat, may become more vulnerable to the reinforcing effects of this drug.

Whether the mediators of cocaine-induced anxiety in HR/LR rats are glucocorticoids, dopaminergic systems, other neuromodulators, or an intricate interplay between them, what is apparent is that experiencing social defeat can profoundly influence subsequent drug-taking behavior and that this effect varies depending on the phenotype of the animal being tested. Differences in novelty seeking, in stress responsiveness, or in sensitivity to the anxietyprovoking features of the drug could explain the behavioral differences in HR and LR rats, both in terms of SA and the impact of social defeat on it.

Finally, the present study suggests that SA of drugs may be secondary to multiple variables, including noveltyseeking behavior in some cases and a somewhat delayed response to social stress in other cases. While significant individual differences exist, social stress appears to be an "equalizer", rendering HR and LR animals similar in terms of their high likelihood of taking drugs.

Acknowledgements This work was supported by NIDA grant numbers DA02265 and DA04294.

\section{References}

Badiani A, Stewart J (1993) Enhancement of the prophagic but not of the antidipsogenic effect of U-50, $488 \mathrm{H}$ after chronic amphetamine. Pharmacol Biochem Behav 44:77-86

Covington HE III, Miczek KA (2001) Repeated social-defeat stress, cocaine or morphine. Effects on behavioral sensitization and intravenous cocaine self-administration "binges". Psychopharmacology DOI 10.1007/s002130100858

Ettenberg A, Geist TD (1991) Animal model for investigating the anxiogenic effects of self-administered cocaine. Psychopharmacology 103:455-461
Goeders NE, Guerin GF (1994) Non-contingent electric footshock facilitates the acquisition of intravenous cocaine self-administration in rats. Psychopharmacology 114:63-70

Goeders NE, Guerin GF (1996) Role of corticosterone in intravenous cocaine self-administration in rats. Neuroendocrinology 64:337-348

Haney M, Miczek KA (1993) Ultrasounds during agonistic interactions between female rats (Rattus norvegicus). J Comp Psychol 107:373-379

Haney M, Maccari S, Le Moal M, Simon H, Piazza PV (1995) Social stress increases the acquisition of cocaine self-administration in male and female rats. Brain Res 698:46-52

Kabbaj M, Devine DP, Savage VR, Akil H (2000) Neurobiological correlates of individual differences in novelty-seeking behavior in the rat: differential expression of stress-related molecules. J Neurosci 20:6983-6988

Kalivas PW, Stewart J (1991) Dopamine transmission in the initiation and expression of drug- and stress-induced sensitization of motor activity. Brain Res Brain Res Rev 16:223-244

Koob GF, Caine SB, Parsons L, Markou A, Weiss F (1997) Opponent process model and psychostimulant addiction. Pharmacol Biochem Behav 57:513-521

Kosten TA, Miserendino MJ, Kehoe P (2000) Enhanced acquisition of cocaine self-administration in adult rats with neonatal isolation stress experience. Brain Res 875:44-50

Marti-Carbonell MA, Darbra S, Garau A, Balada F (1992) Hormones and aggression. Arch Neurobiol (Madr) 55:162-174

Miczek KA (1979) A new test for aggression in rats without aversive stimulation: differential effects of d-amphetamine and cocaine. Psychopharmacology 60:253-259

Miczek KA, Mutschler NH (1996) Activational effects of social stress on IV cocaine self-administration. Psychopharmacology 128:256-264

Miczek KA, Thompson ML, Tornazky W (1991) Subordinate animals: behavioral and physiological adaptations and opioid tolerance. In: Brown MR, Koob GF, Rivier C (eds) Stress: nerobiology and neuroendocrinology. Dekker, New York, pp 323-357

Miczek KA, Weerts E, Haney M, Tidey J (1994) Neurobiological mechanisms controlling aggression: preclinical developments for pharmacotherapeutic interventions. Neurosci Biobehav Rev 18:97-110

Miczek KA, Mutschler NH, van Erp AM, Blank AD, McInerney SC (1999) d-amphetamine "cue" generalizes to social defeat stress: behavioral sensitization and attenuated accumbens dopamine. Psychopharmacology 147:190-199

National Research Council (1996) Guide for the care and use of laboratory animals. National Academy Press, Washington

Piazza PV, Le Moal M (1997) Glucocorticoids as a biological substrate of reward: physiological and pathophysiological implications. Brain Res Brain Res Rev 25:359-372

Piazza PV, Deminiere JM, Le Moal M, Simon H (1989) Factors that predict individual vulnerability to amphetamine selfadministration. Science 245:1511-1513

Piazza PV, Deminiere JM, le Moal M, Simon H (1990) Stress- and pharmacologically-induced behavioral sensitization increases vulnerability to acquisition of amphetamine self-administration. Brain Res 514:22-26

Piazza PV, Maccari S, Deminiere JM, Le Moal M, Mormede P, Simon H (1991) Corticosterone levels determine individual vulnerability to amphetamine self-administration. Proc Natl Acad Sci USA 88:2088-2092

Piazza PV, Deroche V, Rouge-Pont F, Le Moal M (1998) Behavioral and biological factors associated with individual vulnerability to psychostimulant abuse. NIDA Res Monogr 169:105-133

Piazza PV, Deroche-Gamonent V, Rouge-Pont F, Le Moal M (2000) Vertical shifts in self-administration dose-response functions predict a drug-vulnerable phenotype predisposed to addiction. J Neurosci 20:4226-4232

Roberts DC, Loh EA, Vickers G (1989) Self-administration of cocaine on a progressive ratio schedule in rats: dose-response 
relationship and effect of haloperidol pretreatment. Psychopharmacology 97:535-538

Robinson TE, Becker JB (1986) Enduring changes in brain and behavior produced by chronic amphetamine administration: a review and evaluation of animal models of amphetamine psychosis. Brain Res 396:157-198

Rogerio R, Takahashi RN (1992) Anxiogenic properties of cocaine in the rat evaluated with the elevated plus-maze. Pharmacol Biochem Behav 43:631-633

Sarnyai Z (1998) Neurobiology of stress and cocaine addiction. Studies on corticotropin-releasing factor in rats, monkeys, and humans. Ann NY Acad Sci 851:371-387
Sorg BA, Kalivas PW (1991) Effects of cocaine and footshock stress on extracellular dopamine levels in the ventral striatum. Brain Res 559:29-36

Tidey JW, Miczek KA (1996) Social defeat stress selectively alters mesocorticolimbic dopamine release: an in vivo microdialysis study. Brain Res 721:140-149

Tidey JW, Miczek KA (1997) Acquisition of cocaine self-administration after social stress: role of accumbens dopamine. Psychopharmacology 130:203-212

Wise RA (1996) Neurobiology of addiction. Curr Opin Neurobiol $6: 243-251$

Wood DM, Lal H (1987) Anxiogenic properties of cocaine withdrawal. Life Sci 41:1431-1436 